\title{
Approximate Solution of Bagley-Torvik Equations with Variable Coefficients and Three-point Boundary-value Conditions
}

\author{
Q. A. Huang ${ }^{1}$ - X. C. Zhong $^{1}$ • B. L. Guo ${ }^{2}$
}

Published online: 12 May 2015

(C) Springer India Pvt. Ltd. 2015

\begin{abstract}
The fractional Bagley-Torvik equation with variable coefficients is investigated under three-point boundary-value conditions. By using the integration method, the considered problems are transformed into Fredholm integral equations of the second kind. It is found that when the fractional order is $1<\alpha<2$, the obtained Fredholm integral equation is with a weakly singular kernel. When the fractional order is $0<\alpha<1$, the given Fredholm integral equation is with a continuous kernel or a weakly singular kernel depending on the applied boundary-value conditions. The uniqueness of solution for the obtained Fredholm integral equation of the second kind with weakly singular kernel is addressed in continuous function spaces. A new numerical method is further proposed to solve Fredholm integral equations of the second kind with weakly singular kernels. The approximate solution is made and its convergence and error estimate are analyzed. Several numerical examples are computed to show the effectiveness of the solution procedures.
\end{abstract}

Keywords Bagley-Torvik equation - Three-point boundary-value problem . Fredholm integral equation · Weakly singular kernels · Approximate solution

\section{Introduction}

Fractional calculus has profound physics background and rich connotation for mathematical theory and applications [1-3]. In simulating the motion of a rigid plate immersed in a Newtonian fluid, Torvik and Bagley [4] gave a fractional differential equation as

$$
A y^{\prime \prime}(t)+B D^{\alpha} y(t)+C y(t)=F(t), \quad \alpha=\frac{3}{2},
$$

\footnotetext{
$凶 \quad$ X. C. Zhong

xczhong@gxu.edu.cn

1 School of Mathematics and Information Science, Guangxi University, Nanning 530004, Guangxi, People's Republic of China

2 Institute of Applied Physics and Computational Mathematics, Beijing 100088, People's Republic of China
} 
where $A, B$ and $C$ are constants depending on mass and area of the plate, stiffness of spring, fluid density and viscosity. $F(t)$ is a known function denoting the external force. $y(t)$ stands for the displacement of the plate and it should be solved. The Bagley-Torvik equation (1) has been generalized as $\alpha \in(0,2)$ and many methods have been proposed for its general solution and the special solutions with initial value conditions [5-15].

On the other hand, except for initial value problems, one may measure the displacements of the plate at some points and give multipoint boundary value problems of the Bagley-Torvik equation. It is noted that for the generalized nonlinear Bagley-Torvik equation, Stanek [16] has studied the two-point boundary value problem. Moreover, the coefficients $A, B$ and $C$ may also change with the changes of fluid density and viscosity. That is, $A, B$ and $C$ may be functions with respect to $t$. Consequently, here we generally consider the following threepoint boundary value problems of the Bagley-Torvik equation with variable coefficients:

$$
\varphi^{\prime \prime}(x)+p(x) D^{\alpha} \varphi(x)+q(x) \varphi(x)=g(x), \quad 0<\alpha<2, x \in[a, b],
$$

with

$$
\varphi(a)=\alpha_{1}, \quad \varphi(b)+\lambda \varphi(\xi)=\beta_{1}, \quad \xi \in(a, b)
$$

or

$$
\varphi(a)+\mu \varphi(\xi)=\alpha_{1}, \quad \varphi(b)=\beta_{1}, \quad \xi \in(a, b),
$$

where $p(x), q(x)$ and $g(x)$ are known functions. $\alpha_{1}, \beta_{1}, \lambda, \mu$ and $\xi$ are known constants. For convenience, hereafter the fractional derivative $D^{\alpha} \varphi(x)$ is rewritten as ${ }_{a} D_{x}^{\alpha} \varphi(x)$ and we adopt the definition of the Riemann-Liouville fractional derivative, namely

$$
{ }_{a} D_{x}^{\alpha} \varphi(x)=\frac{1}{\Gamma(n-\alpha)} \frac{d^{n}}{d x^{n}} \int_{a}^{x} \frac{\varphi(s)}{(x-s)^{\alpha-n+1}} d s, \quad n-1<\alpha<n,
$$

where $\Gamma$ is the Euler $\gamma$ function.

Furthermore, it is seen that many numerical methods have been given to evaluate fractional order integrals and the solution of fractional order differential equations [17-25]. As shown in the above mentioned works, the principal difficulty in evaluating fractional order integrals is how to deal with the weakly singular kernel. In the typical approaches, the fractional order integrals are approximated by the sum of a discrete convolution and a few correction terms, or the convolution kernel $t^{\alpha-1}(0<\alpha<1)$ is represented as

$$
t^{\alpha-1}=\frac{1}{\Gamma(1-\alpha)} \int_{0}^{\infty} e^{-\xi t} \xi^{-\alpha} d \xi
$$

Furthermore, in numerically solving fractional order differential equations, some methods involving of the main difference method, the Adomian decomposition method, the homotopy function method and the iterative method are proposed. In the present paper, we will transform the three-point boundary value problems for the fractional Bagley-Torvik equation (2) with the boundary-value conditions (3) or (4) into Fredholm integral equations of the second kind. Uniqueness and approximation of the solutions for the obtained Fredholm integral equations will be investigated in detail. Numerical results will carry out to verify the proposed solution procedures.

The paper is organized as follows. In "Fredholm integral equations with weakly singular kernels" section, we transform the boundary value problems (2) with (3) or (4) into a Fredholm integral equation of the second kind by using the integration method. The uniqueness of the solution for the given Fredholm integral equation of the second kind with weakly singular kernel is shown in $\mathbf{C}[a, b]$ by using the contraction operator theorem. "A new numerical method" section shows a new numerical method for solving the obtained Fredholm integral 
equations of the second kind. The approximate solution is made and its convergence and error estimate are considered. Some numerical examples are carried out in "Numerical results" section to show the effectiveness of the proposed methods. "Conclusions" section contains some main conclusions.

\section{Fredholm Integral Equations with Weakly Singular Kernels}

In analyzing multi-point boundary value problems of fractional differential equations, a typical method is to express the solutions as integrals [26-28]. Now let us transform the three-point boundary-value problems (2) with (3) and (4) respectively to Fredholm integral equations of the second kind. It is convenient to define the auxiliary functions as

$$
\begin{aligned}
& V(x, t)= \begin{cases}V_{1}(x, t), & 0<\alpha<1, \\
V_{2}(x, t), & 1 \leq \alpha<2,\end{cases} \\
& \Lambda(x, t)= \begin{cases}\Lambda_{1}(x, t), & 0<\alpha<1, \\
\Lambda_{2}(x, t), & 1 \leq \alpha<2,\end{cases}
\end{aligned}
$$

where

$$
\begin{aligned}
& V_{1}(x, t)=\frac{1}{\Gamma(1-\alpha)}\left[\Gamma(1-\alpha)(x-t) q(t)+\int_{t}^{x} \frac{p(s)-(x-s) p^{\prime}(s)}{(s-t)^{\alpha}} d s\right], \\
& \Lambda_{1}(x, t)=\frac{1}{\Gamma(1-\alpha)}\left[\frac{(\xi-x) p(\xi)}{(\xi-t)^{\alpha}}+\int_{\xi}^{x} \frac{p(s)-(x-s) p^{\prime}(s)}{(s-t)^{\alpha}} d s\right]
\end{aligned}
$$

and

$$
\begin{aligned}
& V_{2}(x, t)=\frac{1}{\Gamma(2-\alpha)}\left[\Gamma(2-\alpha)(x-t) q(t)+\frac{p(x)}{(x-t)^{\alpha-1}}+\int_{t}^{x} \frac{(x-s) p^{\prime \prime}(s)-2 p^{\prime}(s)}{(s-t)^{\alpha-1}} d s\right], \\
& \Lambda_{2}(x, t)=\frac{1}{\Gamma(2-\alpha)}\left[\frac{(x-\xi) p^{\prime}(\xi)-p(\xi)}{(\xi-t)^{\alpha-1}}+\frac{p(x)}{(x-t)^{\alpha-1}}+\int_{\xi}^{x} \frac{(x-s) p^{\prime \prime}(s)-2 p^{\prime}(s)}{(s-t)^{\alpha-1}} d s\right] .
\end{aligned}
$$

\section{The Transformation Method}

Applying the integration method, we give the following two theorems.

Theorem 1 If $(b-a)+\lambda(\xi-a) \neq 0$ and $0<\alpha<2$, the boundary value problem

$$
\left\{\begin{array}{l}
\varphi^{\prime \prime}(x)+p(x) D^{\alpha} \varphi(x)+q(x) \varphi(x)=g(x), \quad x \in[a, b] \\
\varphi(a)=\alpha_{1}, \quad \varphi(b)+\lambda \varphi(\xi)=\beta_{1}, \quad \xi \in(a, b)
\end{array}\right.
$$

is equivalent to the following Fredholm integral equation of the second kind

$$
\varphi(x)+\int_{a}^{b} K_{1}(x, t) \varphi(t) d t=f_{1}(x)
$$


where

$$
\begin{aligned}
& \int \frac{[(b-a)+\lambda(\xi-a)] V(x, t)+(a-x)[V(b, t)+\lambda V(\xi, t)]}{(b-a)+\lambda(\xi-a)}, \\
& \text { for } a \leq t \leq \min \{x, \xi\} \leq b \text {, } \\
& K_{1}(x, t)=\left\{\begin{array}{l}
\frac{(a-x) V(b, t)}{(b-a)+\lambda(\xi-a)}, \text { for } a \leq \max \{x, \xi\} \leq t \leq b, \\
\frac{[(b-a)+\lambda(\xi-a)] V(x, t)+(a-x) V(b, t)}{(b-a)+\lambda(\xi-a)},
\end{array}\right. \\
& \text { for } a \leq \xi \leq t \leq x \leq b, \\
& \frac{(a-x)[V(b, t)+\lambda V(\xi, t)]}{(b-a)+\lambda(\xi-a)}, \text { for } a \leq x \leq t \leq \xi \leq b, \\
& f_{1}(x)=\frac{(b-x)+\lambda(\xi-x)}{(b-a)+\lambda(\xi-a)} \alpha_{1}+\frac{(x-a)}{(b-a)+\lambda(\xi-a)} \beta_{1}+\int_{a}^{b} G_{1}(x, t) g(t) d t,
\end{aligned}
$$

with

$$
G_{1}(x, t)=\left\{\begin{array}{l}
\frac{(t-a)[(x-b)+\lambda(x-\xi)]}{(b-a)+\lambda(\xi-a)}, \quad a \leq t \leq \min \{x, \xi\} \leq b, \\
\frac{(a-x)(b-t)}{(b-a)+\lambda(\xi-a)}, \quad a \leq \max \{x, \xi\} \leq t \leq b, \\
\frac{(b-x)(a-t)+\lambda(\xi-a)(x-t)}{(b-a)+\lambda(\xi-a)}, \quad a \leq \xi \leq t \leq x \leq b, \\
\frac{(a-x)[(b-t)+\lambda(\xi-t)]}{(b-a)+\lambda(\xi-a)}, \quad a \leq x \leq t \leq \xi \leq b .
\end{array}\right.
$$

Proof We first consider the case of $1 \leq \alpha<2$ and integrate both sides of the differential equation in (5) with respect to $x$ from $a$ to $x$ twice. It follows that

$$
\begin{aligned}
& \varphi(x)+\int_{a}^{x}\left\{(x-t) q(t)+\frac{1}{\Gamma(2-\alpha)}\left[\frac{p(x)}{(x-t)^{\alpha-1}}\right.\right. \\
& \left.\left.+\int_{t}^{x} \frac{(x-s) p^{\prime \prime}(s)-2 p^{\prime}(s)}{(s-t)^{\alpha-1}} d s\right]\right\} \varphi(t) d t=\int_{a}^{x}(x-t) g(t) d t \\
& +\left[\left.\frac{p(a)}{\Gamma(2-\alpha)} \frac{d}{d t} \int_{a}^{t} \frac{\varphi(s)}{(t-s)^{\alpha-1}} d s\right|_{t=a}+\varphi^{\prime}(a)\right](x-a)+\varphi(a) .
\end{aligned}
$$

Let $x=b$ in (7) and one has

$$
\begin{aligned}
& \left.\frac{p(a)}{\Gamma(2-\alpha)} \frac{d}{d t} \int_{a}^{t} \frac{\varphi(s)}{(t-s)^{\alpha-1}} d s\right|_{t=a}+\varphi^{\prime}(a) \\
& =\frac{1}{(b-a)}\left[\int_{a}^{b} V_{2}(b, t) \varphi(t) d t+\varphi(b)-\varphi(a)-\int_{a}^{b}(b-t) g(t) d t\right] .
\end{aligned}
$$

From (8) and (7), it is seen that

$$
\begin{aligned}
& \varphi(x)+\int_{a}^{x} V_{2}(x, t) \varphi(t) d t-\int_{a}^{b} \frac{x-a}{b-a} V_{2}(b, t) \varphi(t) d t \\
& =\int_{a}^{x}(x-t) g(t) d t-\int_{a}^{b} \frac{x-a}{b-a}(b-t) g(t) d t \\
& +\frac{b-x}{b-a} \varphi(a)+\frac{x-a}{b-a} \varphi(b) .
\end{aligned}
$$


Then we assume $x=\xi$ in (7) and get

$$
\begin{aligned}
\varphi(x) & +\int_{a}^{x} V_{2}(x, t) \varphi(t) d t-\int_{a}^{\xi} \frac{x-a}{\xi-a} V_{2}(\xi, t) \varphi(t) d t \\
= & \int_{a}^{x}(x-t) g(t) d t-\int_{a}^{\xi} \frac{x-a}{\xi-a}(\xi-t) g(t) d t \\
& +\frac{\xi-x}{\xi-a} \varphi(a)+\frac{x-a}{\xi-a} \varphi(\xi) .
\end{aligned}
$$

With the knowledge of (9), (10) and the boundary conditions in (5), one gives

$$
\begin{gathered}
\varphi(x)+\int_{a}^{x} V_{2}(x, t) \varphi(t) d t+\int_{a}^{b} \frac{(a-x)}{(b-a)+\lambda(\xi-a)} V_{2}(b, t) \varphi(t) d t \\
+\int_{a}^{\xi} \frac{\lambda(a-x)}{(b-a)+\lambda(\xi-a)} V_{2}(\xi, t) \varphi(t) d t=f_{1}(x) .
\end{gathered}
$$

In the end, for various $x, \xi$ and $t$, we can give a series of results. That is,

(1) for $a \leq t \leq \min \{x, \xi\} \leq b$

$$
\begin{aligned}
\varphi(x) & +\int_{a}^{\min \{x, \xi\}} V_{2}(x, t) \varphi(t) d t \\
& +\int_{a}^{\min \{x, \xi\}} \frac{\left[(a-x)\left[V_{2}(b, t)+\lambda V_{2}(\xi, t)\right]\right.}{(b-a)+\lambda(\xi-a)} \varphi(t) d t \\
= & \frac{(b-x)+\lambda(\xi-x)}{(b-a)+\lambda(\xi-a)} \alpha_{1}+\frac{(x-a)}{(b-a)+\lambda(\xi-a)} \beta_{1} \\
& +\int_{a}^{\min \{x, \xi\}} \frac{(t-a)[(x-b)+\lambda(x-\xi)]}{(b-a)+\lambda(\xi-a)} g(t) d t,
\end{aligned}
$$

(2) for $a \leq \max \{x, \xi\} \leq t \leq b$

$$
\begin{aligned}
\varphi(x) & +\int_{\max \{x, \xi\}}^{b} \frac{(a-x) V_{2}(b, t)}{(b-a)+\lambda(\xi-a)} \varphi(t) d t \\
= & \frac{(b-x)+\lambda(\xi-x)}{(b-a)+\lambda(\xi-a)} \alpha_{1}+\frac{(x-a)}{(b-a)+\lambda(\xi-a)} \beta_{1} \\
& +\int_{\max \{x, \xi\}}^{b} \frac{(a-x)(b-t)}{(b-a)+\lambda(\xi-a)} g(t) d t,
\end{aligned}
$$

(3) for $a \leq \xi \leq t \leq x \leq b$

$$
\begin{aligned}
\varphi(x) & +\int_{\xi}^{x} \frac{[(b-a)+\lambda(\xi-a)] V_{2}(x, t)+(a-x) V_{2}(b, t)}{(b-a)+\lambda(\xi-a)} \varphi(t) d t \\
= & \frac{(b-x)+\lambda(\xi-x)}{(b-a)+\lambda(\xi-a)} \alpha_{1}+\frac{(x-a)}{(b-a)+\lambda(\xi-a)} \beta_{1} \\
& +\int_{\xi}^{x} \frac{(b-x)(a-t)+\lambda(\xi-a)(x-t)}{(b-a)+\lambda(\xi-a)} g(t) d t,
\end{aligned}
$$


(4) for $a \leq x<t<\xi \leq b$

$$
\begin{aligned}
\varphi(x) & +\int_{x}^{\xi} \frac{(a-x)\left[V_{2}(b, t)+\lambda V_{2}(\xi, t)\right]}{(b-a)+\lambda(\xi-a)} \varphi(t) d t \\
= & \frac{(b-x)+\lambda(\xi-x)}{(b-a)+\lambda(\xi-a)} \alpha_{1}+\frac{(x-a)}{(b-a)+\lambda(\xi-a)} \beta_{1} \\
& +\int_{x}^{\xi} \frac{(a-x)[(b-t)+\lambda(\xi-t)]}{(b-a)+\lambda(\xi-a)} g(t) d t .
\end{aligned}
$$

So the theorem is proved after rewriting (12)-(15) as (6). Similarly, for the case of $0<\alpha<1$, we can transform the three-point boundary value problem (5) into Fredholm integral equation of the second kind. The detail procedures are omitted here for saving spaces.

Theorem 2 If $(b-a)+\mu(b-\xi) \neq 0$ and $0<\alpha<2$, the boundary value problem

$$
\left\{\begin{array}{l}
\varphi^{\prime \prime}(x)+p(x) D^{\alpha} \varphi(x)+q(x) \varphi(x)=g(x), \quad x \in[a, b], \\
\varphi(a)+\mu \varphi(\xi)=\alpha_{1}, \quad \varphi(b)=\beta_{1}, \quad \xi \in(a, b),
\end{array}\right.
$$

is equivalent to the following Fredholm integral equation of the second kind

$$
\varphi(x)+\int_{a}^{b} K_{2}(x, t) \varphi(t) d t=f_{2}(x)
$$

where

$$
\begin{aligned}
& K_{2}(x, t)=\left\{\begin{array}{l}
\frac{[(b-a) V(x, t)+(a-x) V(b, t)]+\mu[(b-\xi) \Lambda(x, t)+(\xi-x) \Lambda(b, t)]}{(b-a)+\mu(b-\xi)}, \\
\quad \text { for } a \leq t \leq \min \{x, \xi\} \leq b, \\
\frac{(a-x)+\mu(\xi-x)}{(b-a)+\mu(b-\xi)} V(b, t), \quad \text { for } a \leq \max \{x, \xi\} \leq t \leq b, \\
\frac{[(b-a) V(x, t)+(a-x) V(b, t)]+\mu[(b-\xi) V(x, t)+(\xi-x) V(b, t)]}{(b-a)+\mu(b-\xi)}, \\
\quad \text { for } a \leq \xi \leq t \leq x \leq b, \\
\frac{[(a-x) V(b, t)+\mu(\xi-x) \Lambda(b, t)]+\mu(\xi-b)[V(x, t)-\Lambda(x, t)]}{(b-a)+\mu(b-\xi)}, \\
\text { for } a \leq x \leq t \leq \xi \leq b,
\end{array}\right. \\
& f_{2}(x)=\frac{(b-x)}{(b-a)+\mu(b-\xi)} \alpha_{1}+\frac{(x-a)+\mu(x-\xi)}{(b-a)+\mu(b-\xi)} \beta_{1}+\int_{a}^{b} G_{2}(x, t) g(t) d t,
\end{aligned}
$$

with

$$
G_{2}(x, t)=\left\{\begin{array}{l}
\frac{(b-x)(a-t)}{(b-a)+\mu(b-\xi)}, \quad a \leq t \leq \min \{x, \xi\} \leq b, \\
\frac{(a-x)+\mu(\xi-x)}{(b-a)+\mu(b-\xi)}(b-t), \quad a \leq \max \{x, \xi\} \leq t \leq b, \\
\frac{(b-x)[(a-t)+\mu(\xi-t)]}{(b-a)+\mu(b-\xi)}, \quad a \leq \xi \leq t \leq x \leq b, \\
\frac{(a-x)(b-t)+\mu(\xi-b)(x-t)}{(b-a)+\mu(b-\xi)}, \quad a \leq x \leq t \leq \xi \leq b .
\end{array}\right.
$$


Proof For $1 \leq \alpha<2$, we integrate both sides of the differential equation in (16) with respect to $x$ from $\xi$ to $x$ twice, and arrive at

$$
\begin{aligned}
\varphi(x) & +\int_{a}^{x}\left[\frac{1}{\Gamma(2-\alpha)} \frac{p(x)}{(x-t)^{\alpha-1}}\right] \varphi(t) d t \\
& +\int_{\xi}^{x}\left[(x-t) q(t)+\frac{1}{\Gamma(2-\alpha)} \int_{t}^{x} \frac{(x-s) p^{\prime \prime}(s)-2 p^{\prime}(s)}{(s-t)^{\alpha-1}} d s\right] \varphi(t) d t \\
& +\int_{a}^{\xi} \frac{1}{\Gamma(2-\alpha)}\left[\frac{(x-\xi) p^{\prime}(\xi)-p(\xi)}{(\xi-t)^{\alpha-1}}+\int_{\xi}^{x} \frac{(x-s) p^{\prime \prime}(s)-2 p^{\prime}(s)}{(s-t)^{\alpha-1}} d s\right] \varphi(t) d t \\
= & \int_{\xi}^{x}(x-t) g(t) d t+\left[\left.\frac{p(\xi)}{\Gamma(2-\alpha)} \frac{d}{d t} \int_{a}^{t} \frac{\varphi(s)}{(t-s)^{\alpha-1}} d s\right|_{t=\xi}+\varphi^{\prime}(\xi)\right](x-\xi)+\varphi(\xi),
\end{aligned}
$$

or

$$
\begin{gathered}
\varphi(x)+\int_{\xi}^{x} V_{2}(x, t) \varphi(t) d t+\int_{a}^{\xi} \Lambda_{2}(x, t) \varphi(t) d t=\int_{\xi}^{x}(x-t) g(t) d t \\
+\left[\left.\frac{p(\xi)}{\Gamma(2-\alpha)} \frac{d}{d t} \int_{a}^{t} \frac{\varphi(s)}{(t-s)^{\alpha-1}} d s\right|_{t=\xi}+\varphi^{\prime}(\xi)\right](x-\xi)+\varphi(\xi) .
\end{gathered}
$$

Assume $x=b$ in (19) and one has

$$
\begin{gathered}
\left.\frac{p(\xi)}{\Gamma(2-\alpha)} \frac{d}{d t} \int_{a}^{t} \frac{\varphi(s)}{(t-s)^{\alpha-1}} d s\right|_{t=\xi}+\varphi^{\prime}(\xi)=\frac{1}{(b-\xi)}[\varphi(b)-\varphi(\xi) \\
\left.-\int_{\xi}^{b}(b-t) g(t) d t+\int_{a}^{\xi} \Lambda_{2}(b, t) \varphi(t) d t+\int_{\xi}^{b} V_{2}(b, t) \varphi(t) d t\right] .
\end{gathered}
$$

According to (19) and (20), it is found that

$$
\begin{aligned}
\varphi(x) & +\int_{\xi}^{x} V_{2}(x, t) \varphi(t) d t-\frac{x-\xi}{b-\xi} \int_{\xi}^{b} V_{2}(b, t) \varphi(t) d t \\
& +\int_{a}^{\xi} \Lambda_{2}(x, t) \varphi(t) d t-\frac{x-\xi}{b-\xi} \int_{a}^{\xi} \Lambda_{2}(b, t) \varphi(t) d t \\
& =\int_{\xi}^{x}(x-t) g(t) d t-\frac{x-\xi}{b-\xi} \int_{\xi}^{b}(b-t) g(t) d t+\frac{b-x}{b-\xi} \varphi(\xi)+\frac{x-\xi}{b-\xi} \varphi(b) .
\end{aligned}
$$

Making use of the boundary conditions in (16), one can obtain

$$
\begin{gathered}
\varphi(x)+\int_{a}^{x} \frac{(b-a) V_{2}(x, t)}{(b-a)+\mu(b-\xi)} \varphi(t) d t+\int_{a}^{b} \frac{(a-x) V_{2}(b, t)}{(b-a)+\mu(b-\xi)} \varphi(t) d t \\
+\int_{\xi}^{x} \frac{\mu(b-\xi) V_{2}(x, t)}{(b-a)+\mu(b-\xi)} \varphi(t) d t+\int_{\xi}^{b} \frac{\mu(\xi-x) V_{2}(b, t)}{(b-a)+\mu(b-\xi)} \varphi(t) d t \\
+\int_{a}^{\xi} \frac{\mu\left[(b-\xi) \Lambda_{2}(x, t)+(\xi-x) \Lambda_{2}(b, t)\right]}{(b-a)+\mu(b-\xi)} \varphi(t) d t=f_{2}(x) .
\end{gathered}
$$

Similar to Theorem 1, we can rewrite (22) as (17) by considering the various values of $x, \xi$ and $t$. Furthermore, the results for $0<\alpha<1$ can be obtained and the theorem is proved. 
It is seen from Theorems 1 and 2 that one has transformed the three-point boundary value problems for the generalized Bagley-Torvik equation into Fredholm integral equations of the second kind. It is interesting to find that the kernel $K_{1}(x, t)$ is continuous for $\alpha \in$ $(0,1)$ and weakly singular for $\alpha \in(1,2)$, and the kernel $K_{2}(x, t)$ is weakly singular for $\alpha \in(0,1) \cup(1,2)$. In addition, when $\alpha=1$, corresponding to three-point boundary value problems of second-order ordinary differential equations, the obtained results in Theorems 1 and 2 can be degenerated to those in [29].

\section{Uniqueness of the Solution}

In the subsection, let us focus on the uniqueness of the solutions of the obtained Fredholm integral equations (6) and (17) in the space $\mathbf{C}[a, b]$. When the derived Fredholm integral equation is with the continuous kernel, the uniqueness theorem of its solution can be given similar to those in [29] and the detail procedure is omitted here. When the derived Fredholm integral equation is with the weakly singular kernel, the uniqueness of its solution will be investigated in detail. One can see that the uniqueness of the solution for Fredholm integral equations with weakly singular kernels has been investigated comprehensively [30, 31]. However, the kernels in (6) and (17) are different from the typical weakly singular kernels, and it is significant to address the uniqueness of the solution for the obtained Fredholm integral equations with weakly singular kernels. In what follows, according to the contraction operator theorem in continuous function spaces, the theorem about the uniqueness of the solution will be given.

Theorem 3 It is assumed that $1<\alpha<2$ and $p(x) \in \mathbf{C}^{2}[a, b], q(x) \in \mathbf{L}^{1}[a, b], g(x) \in$ $\mathbf{L}^{1}[a, b]$ for finite $a$ and $b$. When

$$
\max _{a \leq x \leq b} \int_{a}^{b}\left|K_{i}(x, t)\right| d t<1, \quad i=1,2,
$$

the Fredholm integral equations of the second kind (6) and (17) have unique solutions in $\mathbf{C}[a, b]$.

Proof For convenience, Eq. (6) is written as

$$
\varphi=f_{1}-\mathbf{K} \varphi=\mathbf{T} \varphi
$$

with

$$
\mathbf{K} \varphi=\int_{a}^{b} K_{1}(x, t) \varphi(t) d t
$$

It is seen that the operator $\mathbf{K}: \mathbf{C}[a, b] \rightarrow \mathbf{C}[a, b]$ is linear and bounded. In fact, since $p(x) \in \mathbf{C}^{2}[a, b], q(x) \in \mathbf{L}^{1}[a, b],(x-t) \in \mathbf{C}[a, b], 1 /(x-t)^{\alpha-1} \in \mathbf{L}^{1}[a, b]$ and $a$ and $b$ are finite, it is obtained that $V_{2}(x, t), \Lambda_{2}(x, t) \in \mathbf{L}^{1}[a, b ; a, b]$. Hence one gets $K_{1}(x, t) \in$ $\mathbf{L}^{1}[a, b ; a, b]$. Furthermore, we let

$$
\max _{a \leq x \leq b} \int_{a}^{b}\left|K_{1}(x, t)\right| d t=c, \quad c \geq 0 .
$$

For $\varphi(x) \in \mathbf{C}[a, b]$, it gives

$$
\|\mathbf{K} \varphi\|_{\infty}=\left\|\int_{a}^{b} K_{1}(x, t) \varphi(t) d t\right\|_{\infty} \leq \max _{a \leq x \leq b} \int_{a}^{b}\left|K_{1}(x, t)\right| d t \cdot\|\varphi\|_{\infty}=c\|\varphi\|_{\infty} .
$$


So the operator $\mathbf{K}$ is linear and bounded, and

$$
\|\mathbf{K}\|_{\infty} \leq \max _{a \leq x \leq b} \int_{a}^{b}\left|K_{1}(x, t)\right| d t=c .
$$

For all $\varphi_{1}, \varphi_{2} \in \mathbf{C}$, one has

$$
\left\|\mathbf{T} \varphi_{1}-\mathbf{T} \varphi_{2}\right\|_{\infty}=\left\|\mathbf{K}\left(\varphi_{1}-\varphi_{2}\right)\right\|_{\infty} \leq\|\mathbf{K}\|_{\infty} \cdot\left\|\varphi_{1}-\varphi_{2}\right\|_{\infty} \leq c\left\|\varphi_{1}-\varphi_{2}\right\|_{\infty} .
$$

When $c<1$, the Fredholm integral equation of the second kind (6) has a unique solution in $\mathbf{C}[a, b]$ by using Banach fixed point theorem. Similarly, for Eq. (17), it is easy to prove that there is a unique solution in $\mathbf{C}[a, b]$. This completes the proof.

Moreover, we have the following theorem.

Theorem 4 Under the assumption of $0<\alpha<1$ and $p(x) \in \mathbf{C}[a, b], q(x) \in \mathbf{L}^{1}[a, b]$, $g(x) \in \mathbf{L}^{1}[a, b]$ for finite $a$ and $b$, the Fredholm integral equations of the second kind (6) and (17) have unique solutions in $\mathbf{C}[a, b]$ when

$$
\max _{a \leq x \leq b} \int_{a}^{b}\left|K_{i}(x, t)\right| d t<1, \quad i=1,2 .
$$

The proof of Theorem 4 is similar to that of Theorem 3 and it has been omitted here. From Theorems (3) and (4), it is seen that one only gives the sufficient conditions for the uniqueness of solution in $\mathbf{C}[a, b]$. The applied method is different from those in [30,31], where the iterative method is applied. The reason is based on the fact that the kernels in Eqs. (6) and (17) are different from the typical weakly singular kernel $1 /|x-t|$. They have the following style

$$
K(x, t)=\frac{A_{0}(x, t)}{(x-t)^{\alpha_{0}}}+\frac{B_{0}(x, t)}{(b-t)^{\alpha_{0}}}+\frac{C_{0}(x, t)}{(\xi-t)^{\alpha_{0}}},
$$

with $0<\alpha_{0}<1$ and the piecewise continuous functions $A_{0}(x, t), B_{0}(x, t)$ and $C_{0}(x, t)$.

\section{A New Numerical Method}

In what follows, we turn our attention to the numerical solutions of Eqs. (6) and (17). Recently, the piecewise Taylor-series expansion method is proposed to solve Fredholm integral equations of the second kind [29]. Unfortunately, the kernels in Eqs. (6) and (17) may be weakly singular, then the proposed method in [29] cannot be used directly. Consequently, we will modify the numerical method in [29] so that it can be used to solve Fredholm integral equations of the second kind with weakly singular kernels.

\section{Construction of the Approximate Solution}

Generally Fredholm integral equations of the second kind can be written as:

$$
\varphi(x)+\int_{a}^{b} K(x, t) \varphi(t) d t=f(x), \quad x \in[a, b],
$$

where $f(x)$ is a known function on the finite interval $[a, b] . \varphi(x)$ is unknown to be solved, and the kernel $K(x, t)$ is weakly singular. Different from the differential method in [29], we 
integrate both sides of Eq. (23) $i(i=1,2, \ldots, n)$ times and get

$$
\begin{aligned}
& \int_{a}^{x} \frac{(x-t)^{i-1}}{(i-1) !} \varphi(t) d t+\int_{a}^{x} \frac{(x-s)^{i-1}}{(i-1) !}\left[\int_{a}^{b} K(s, t) \varphi(t) d t\right] d s \\
& =\int_{a}^{x} \frac{(x-t)^{i-1}}{(i-1) !} f(t) d t .
\end{aligned}
$$

Exchanging the integral order in (24), one has

$$
\begin{aligned}
& \int_{a}^{x} \frac{(x-t)^{i-1}}{(i-1) !} \varphi(t) d t+\int_{a}^{b}\left[\int_{a}^{x} \frac{(x-s)^{i-1}}{(i-1) !} K(s, t) d s\right] \varphi(t) d t \\
& \quad=\int_{a}^{x} \frac{(x-t)^{i-1}}{(i-1) !} f(t) d t .
\end{aligned}
$$

Similar to those in [29], we choose a series of quadrature points such as

$$
a=x_{0}<x_{1}<\cdots<x_{m}=b \quad(m \geq 1)
$$

where $x_{q}=a+q h \quad(q=0,1, \ldots, m)$ with $h=(b-a) / m$. Letting $x=x_{k}(k=$ $1,2, \ldots, m)$, Eq. (25) can be further written as

$$
\begin{aligned}
& \sum_{q=0}^{k-1} \int_{x_{q}}^{x_{q+1}} \frac{\left(x_{k}-t\right)^{i-1}}{(i-1) !} \varphi(t) d t+\sum_{q=0}^{m-1} \sum_{p=0}^{k-1} \int_{x_{q}}^{x_{q+1}}\left[\int_{x_{p}}^{x_{p+1}} \frac{\left(x_{k}-s\right)^{i-1}}{(i-1) !} K(s, t) d s\right] \varphi(t) d t \\
& \quad=\sum_{q=0}^{k-1} \int_{x_{q}}^{x_{q+1}} \frac{\left(x_{k}-t\right)^{i-1}}{(i-1) !} f(t) d t .
\end{aligned}
$$

Furthermore we let $t=x_{q}+h \eta$ and $s=x_{p}+h \zeta$, Eq. (26) can be written as

$$
\begin{aligned}
& \sum_{q=0}^{k-1} \frac{h^{i}}{(i-1) !} \int_{0}^{1}(k-q-\eta)^{i-1} \varphi\left(x_{q}+h \eta\right) d \eta+ \\
& \sum_{q=0}^{m-1} \sum_{p=0}^{k-1} \frac{h^{i+1}}{(i-1) !} \int_{0}^{1} \int_{0}^{1}(k-p-\zeta)^{i-1} K\left(x_{p}+h \zeta, x_{q}+h \eta\right) \varphi\left(x_{q}+h \eta\right) d \zeta d \eta \\
& \quad=\sum_{q=0}^{k-1} \frac{h^{i}}{(i-1) !} \int_{0}^{1}(k-q-\eta)^{i-1} f\left(x_{q}+h \eta\right) d \eta .
\end{aligned}
$$

It is assumed that $\varphi\left(x_{q}+h \eta\right)$ can be expressed as a Taylor-series expansion with Lagrange remainder. That is, we have

$$
\varphi\left(x_{q}+h \eta\right)=\sum_{j=0}^{n} \frac{\varphi^{(j)}\left(x_{q}\right)}{j !}(h \eta)^{j}+\frac{\varphi^{(n+1)}\left(\theta_{q}\right)}{(n+1) !}(h \eta)^{n+1}, \quad x_{q} \leq \theta_{q} \leq x_{q}+h \eta .
$$


Insertion of Eq. (28) into Eq. (27) yields

$$
\begin{aligned}
& \sum_{q=0}^{k-1} \frac{h^{i}}{(i-1) !} \int_{0}^{1}(k-q-\eta)^{i-1}\left[\sum_{j=0}^{n} \frac{\varphi^{(j)}\left(x_{q}\right)}{j !}(h \eta)^{j}+\frac{\varphi^{(n+1)}\left(\theta_{q}\right)}{(n+1) !}(h \eta)^{n+1}\right] d \eta \\
& \quad+\sum_{q=0}^{m-1} \sum_{p=0}^{k-1} \frac{h^{i+1}}{(i-1) !} \int_{0}^{1} \int_{0}^{1}(k-p-\zeta)^{i-1} K\left(x_{p}+h \zeta, x_{q}+h \eta\right) \\
& {\left[\sum_{j=0}^{n} \frac{\varphi^{(j)}\left(x_{q}\right)}{j !}(h \eta)^{j}+\frac{\varphi^{(n+1)}\left(\theta_{q}\right)}{(n+1) !}(h \eta)^{n+1}\right] d \zeta d \eta} \\
& \quad \sum_{q=0}^{k-1} \frac{h^{i}}{(i-1) !} \int_{0}^{1}(k-q-\eta)^{i-1} f\left(x_{q}+h \eta\right) d \eta .
\end{aligned}
$$

In Eq. (29), one replaces $\varphi^{(j)}\left(x_{q}\right)$ by the numerical solution $\varphi_{q}^{(j)}$ and obtains a discrete format as

$$
\begin{aligned}
& \sum_{q=0}^{k-1} \sum_{j=0}^{n} \frac{\varphi_{q}^{(j)} h^{i+j}}{(i-1) ! j !} \int_{0}^{1}(k-q-\eta)^{i-1} \eta^{j} d \eta+ \\
& \sum_{q=0}^{m-1} \sum_{p=0}^{k-1} \sum_{j=0}^{n} \frac{\varphi_{q}^{(j)} h^{i+j+1}}{(i-1) ! j !} \int_{0}^{1} \int_{0}^{1}(k-p-\zeta)^{i-1} K\left(x_{p}+h \zeta, x_{q}+h \eta\right) \eta^{j} d \zeta d \eta \\
& \quad=\sum_{q=0}^{k-1} \frac{h^{i}}{(i-1) !} \int_{0}^{1}(k-q-\eta)^{i-1} f\left(x_{q}+h \eta\right) d \eta .
\end{aligned}
$$

where $i=1,2, \ldots, n$ and $k=1,2, \ldots, m$. Moreover, applying Eqs. (28) to (23), Eq. (23) can be approximated by the following discrete formation

$$
\varphi_{k-1}^{(0)}+\sum_{q=0}^{m-1} \sum_{j=0}^{n} \frac{\varphi_{q}^{(j)} h^{j+1}}{j !} \int_{0}^{1} K\left(x_{k-1}, x_{q}+h \eta\right) \eta^{j} d \eta=f\left(x_{k-1}\right)
$$

for $q=0,1, \ldots,(m-1)$. Once the linear system of (30) and (31) with $m(n+1)$ equations is solved, the approximate solution of $\varphi(x)$ can be further given as

$$
\varphi_{m, n}(x)=f(x)-\sum_{q=0}^{m-1} \sum_{j=0}^{n} \frac{\varphi_{q}^{(j)} h^{j+1}}{j !} \int_{0}^{1} K\left(x, x_{q}+h \eta\right) \eta^{j} d \eta, \quad a \leq x \leq b .
$$

\section{Convergence and Error Estimate}

Now we focus on the convergence and error estimate of the approximate solution $\varphi_{m, n}(x)$. First, we rewrite Eqs. (30) and (31) as

$$
(A+B) \tilde{\Phi}=F
$$


where

$$
\begin{aligned}
& A=\left[a_{s t}\right]_{m(n+1) \times m(n+1)}, \\
& B=\left[b_{s t}\right]_{m(n+1) \times m(n+1)} \\
& F=F_{m(n+1) \times 1}=\left[f\left(x_{0}\right), f\left(x_{1}\right), \ldots, \sum_{q=0}^{m-1} \frac{h^{n}}{(n-1) !} \int_{0}^{1}(m-q-\eta)^{n-1} f\left(x_{q}+h \eta\right) d \eta\right] \\
& \tilde{\Phi}=\left[\varphi_{q}^{(j)}\right]_{m(n+1) \times 1}=\left[\varphi_{0}^{(0)}, \varphi_{1}^{(0)}, \ldots, \varphi_{m-1}^{(n)}\right]^{T},
\end{aligned}
$$

with

$$
\begin{aligned}
& a_{11}=1 \text {, } \\
& a_{12}=0 \text {, } \\
& a_{m(n+1), m(n+1)}=\frac{h^{2 n}}{(n-1) ! n !} \int_{0}^{1}(1-\eta)^{n-1} \eta^{n} d \eta, \\
& b_{11}=h \int_{0}^{1} K\left(x_{0}, x_{0}+h \eta\right) d \eta \text {, } \\
& b_{12}=h \int_{0}^{1} K\left(x_{0}, x_{1}+h \eta\right) d \eta \text {, } \\
& b_{m(n+1), m(n+1)}=\sum_{p=0}^{m-1} \frac{h^{2 n+1}}{(n-1) ! n !} \int_{0}^{1} \int_{0}^{1}(m-p-\zeta)^{n-1} K\left(x_{p}+h \zeta, x_{m-1}+h \eta\right) \eta^{n} d \zeta d \eta .
\end{aligned}
$$

Moreover, by considering the Lagrange remainder, it follows that

$$
(A+B) \Phi=F-R,
$$

where

$$
\begin{aligned}
\Phi & =\left[\varphi^{(j)}\left(x_{q}\right)\right]_{m(n+1) \times 1}=\left[\varphi^{(0)}\left(x_{0}\right), \varphi^{(0)}\left(x_{1}\right), \cdots, \varphi^{(n)}\left(x_{m-1}\right)\right]^{T}, \\
R & =\left[r_{s}\right]_{m(n+1) \times 1},
\end{aligned}
$$

with

$$
\begin{aligned}
& r_{1}=\sum_{q=0}^{m-1} \frac{h^{n+2}}{(n+1) !} \int_{0}^{1} K\left(x_{0}, x_{q}+h \eta\right) \varphi^{(n+1)}\left(\theta_{q}\right) \eta^{n+1} d \eta, \\
& r_{2}=\sum_{q=0}^{m-1} \frac{h^{n+2}}{(n+1) !} \int_{0}^{1} K\left(x_{1}, x_{q}+h \eta\right) \varphi^{(n+1)}\left(\theta_{q}\right) \eta^{n+1} d \eta, \\
& \ldots \ldots \ldots \ldots \\
& r_{m(n+1)}=\sum_{q=0}^{m-1} \frac{h^{2 n+1}}{(n-1) !(n+1) !} \int_{0}^{1}(m-q-\eta)^{n-1} \varphi^{(n+1)}\left(\theta_{q}\right) \eta^{n+1} d \eta+\sum_{q=0}^{m-1} \sum_{p=0}^{m-1} \\
& \quad \cdot \frac{h^{2 n+2}}{(n-1) !(n+1) !} \int_{0}^{1} \int_{0}^{1}(m-p-\zeta)^{n-1} K\left(x_{p}+h \zeta, x_{q}+h \eta\right) \varphi^{(n+1)}\left(\theta_{q}\right) \eta^{n+1} d \zeta d \eta .
\end{aligned}
$$


It is seen that the matrix $A$ is nonsingular, and one lets

$$
\mathcal{A}: \mathbf{R}^{m(n+1)} \rightarrow \mathbf{R}^{m(n+1)}
$$

be a mapping. Given $x \in \mathbf{R}^{m(n+1)}$ we definite

$$
\mathcal{A} x=A x .
$$

It is easy to prove that $\mathcal{A}$ is a linear operator, and $\mathcal{A}$ is bijective. In addition, one can calculate that

$$
\begin{aligned}
\|A\|_{\infty} & =\max \left\{1, \max _{1 \leq k \leq m, 1 \leq i \leq n} \sum_{j=0}^{n} \sum_{q=0}^{k-1} \frac{h^{i+j}}{(i-1) ! j !}\left|\int_{0}^{1}(k-q-\eta)^{i-1} \eta^{j} d \eta\right|\right\} \\
& \leq \max \left\{1, \max _{1 \leq k \leq m, 1 \leq i \leq n} \sum_{j=0}^{n} \sum_{q=0}^{k-1} \frac{h^{i+j}}{(i-1) ! j !} \int_{0}^{1}(k-q-\eta)^{i-1} d \eta\right\} \\
& =\max \left\{1, \sum_{j=0}^{n} \frac{h^{j}}{j !} \cdot \max _{1 \leq k \leq m, 1 \leq i \leq n} \frac{h^{i}}{i !} \sum_{q=0}^{k-1}\left[(k-q)^{i}-(k-q-1)^{i}\right]\right\} \\
& \leq \max \left\{1, \sum_{j=0}^{+\infty} \frac{h^{j}}{j !} \cdot \max _{1 \leq k \leq m, 1 \leq i \leq n} \frac{(h k)^{i}}{i !}\right\} \leq \max \left\{1, e^{h} \cdot \sum_{i=1}^{n} \frac{(b-a)^{i}}{i !}\right\} \\
& \leq \max \left\{1, e^{h+b-a}\right\} \leq e^{h+b-a} \leq e^{2(b-a)}<+\infty .
\end{aligned}
$$

So $\mathcal{A}$ is a bounded operator in $\mathbf{R}^{m(n+1)}$. According to the inverse operator theorem in Banach spaces [32], the inverse operator $\mathcal{A}^{-1}$ is bounded. Now we give the following theorem:

Theorem 5 We let $\left\|A^{-1}\right\|_{\infty} \leq L$ and have the following conditions

$$
\begin{aligned}
& \max _{q=0,1, \cdots, m-1} \max _{a \leq x \leq b} \int_{0}^{1}\left|K\left(x, x_{q}+h \eta\right)\right| d \eta=M<\frac{1}{L(b-a) e^{h+(b-a)}}<+\infty, \\
& \left\|\varphi^{(n+1)}(x)\right\|_{\infty}=\max _{a \leq x \leq b}\left|\varphi^{(n+1)}(x)\right|=N<+\infty .
\end{aligned}
$$

The approximate solution $\varphi_{m, n}(x)$ in (32) is convergent to the exact solution $\varphi(x)$. That is, we get

$$
\lim _{n \rightarrow+\infty}\left\|\varphi_{m, n}(x)-\varphi(x)\right\|_{\infty}=0
$$

and

$$
\lim _{m \rightarrow+\infty}\left\|\varphi_{m, n}(x)-\varphi(x)\right\|_{\infty}=0 .
$$

Moreover, the following error estimate can be obtained

$$
\left\|\varphi_{m, n}(x)-\varphi(x)\right\|_{\infty} \leq \frac{(b-a) M N\left[1+L h e^{h+(b-a)}\right]}{\left[1-(b-a) M L e^{h+(b-a)}\right]} \cdot \frac{h^{n+1}}{(n+1) !} .
$$


Proof One can calculate that

$$
\begin{aligned}
&\|B\|_{\infty}=\max \left\{\max _{1 \leq k \leq m} \sum_{j=0}^{n} \sum_{q=0}^{m-1} \frac{h^{j+1}}{j !}\left|\int_{0}^{1} K\left(x_{k-1}, x_{q}+h \eta\right) \eta^{j} d \eta\right|,\right. \\
& \max _{1 \leq k \leq m, 1 \leq i \leq n} \sum_{j=0}^{n} \sum_{q=0}^{m-1} \sum_{p=0}^{k-1} \frac{h^{i+j+1}}{(i-1) ! j !} \\
&\left.\cdot\left|\int_{0}^{1} \int_{0}^{1}(k-p-\zeta)^{i-1} K\left(x_{p}+h \zeta, x_{q}+h \eta\right) \eta^{j} d \zeta d \eta\right|\right\} \\
& \leq \max \left\{h m M e^{h}, h m M \sum_{j=0}^{n} \frac{h^{j}}{j !} \cdot \max _{1 \leq k \leq m, 1 \leq i \leq n} \frac{h^{i}}{(i-1) !} \sum_{p=0}^{k-1} \int_{0}^{1}(k-p-\zeta)^{i-1} d \zeta\right\} \\
& \leq \max \left\{h m M e^{h}, h m M e^{h} \cdot \sum_{i=1}^{n} \frac{(b-a)^{i}}{i !}\right\} \leq(b-a) M e^{h+(b-a)},
\end{aligned}
$$

and

$$
\begin{aligned}
\|R\|_{\infty}= & \max \left\{\max _{1 \leq k \leq m}\left|\sum_{q=0}^{m-1} \frac{h^{n+2}}{(n+1) !} \int_{0}^{1} K\left(x_{k-1}, x_{q}+h \eta\right) \varphi^{(n+1)}\left(\theta_{q}\right) \eta^{n+1} d \eta\right|,\right. \\
& \max _{1 \leq k \leq m, 1 \leq i \leq n} \mid \sum_{q=0}^{k-1} \frac{h^{i+n+2}}{(i-1) !(n+1) !} \int_{0}^{1}(k-q-\eta)^{i-1} \varphi^{(n+1)}\left(\theta_{q}\right) \eta^{n+1} d \eta \\
& +\sum_{q=0}^{m-1} \sum_{p=0}^{k-1} \frac{h^{i+n+2}}{(i-1) !(n+1) !} \int_{0}^{1} \int_{0}^{1}(k-p-\zeta)^{i-1} K\left(x_{p}+h \zeta, x_{q}+h \eta\right) \\
& \left.\times \varphi^{(n+1)}\left(\theta_{q}\right) \eta^{n+1} d \zeta d \eta \mid\right\} \\
\leq & \max \left\{\frac{(b-a) M N h^{n+1}}{(n+1) !}, \frac{N h^{n+2}}{(n+1) !} e^{b-a}+\frac{(b-a) M N h^{n+1}}{(n+1) !} e^{b-a}\right\} \\
\leq & \frac{N h^{n+1} e^{b-a}[h+(b-a) M]}{(n+1) !} .
\end{aligned}
$$

Then one has $\left\|B A^{-1}\right\|_{\infty} \leq\|B\|_{\infty}\left\|A^{-1}\right\|_{\infty}<1$. It is easy to prove that $I+B A^{-1}$ is a strictly diagonally dominant matrix, where $I$ is a unity matrix. Hence, it is found that $I+B A^{-1}$ is a nonsingular matrix. Furthermore, one can calculate that

$$
\begin{aligned}
\| & \Phi-\tilde{\Phi}\left\|_{\infty}=\right\|\left[\left(I+B A^{-1}\right) A\right]^{-1} R \|_{\infty} \\
& \leq\left\|A^{-1}\right\|_{\infty}\left\|\left(I+B A^{-1}\right)^{-1}\right\|_{\infty}\|R\|_{\infty} \\
& \leq \frac{\left\|A^{-1}\right\|_{\infty}\|R\|_{\infty}}{1-\left\|B A^{-1}\right\|_{\infty}} \leq \frac{\left\|A^{-1}\right\|_{\infty}\|R\|_{\infty}}{1-\|B\|_{\infty}\left\|A^{-1}\right\|_{\infty}} \\
& \leq \frac{N L h^{n+1} e^{b-a}[h+(b-a) M]}{\left[1-(b-a) M L e^{h+(b-a)}\right](n+1) !} .
\end{aligned}
$$


It is easy to obtain

$$
\begin{aligned}
\left\|\varphi(x)-\varphi_{m, n}(x)\right\|_{\infty} & \left\|\sum_{q=0}^{m-1} h \int_{0}^{1} K\left(x, x_{q}+h \eta\right)\left[\varphi\left(x_{q}+h \eta\right)-\sum_{j=0}^{n} \frac{\varphi_{q}^{(j)} h^{j} \eta^{j}}{j !}\right] d \eta\right\|_{\infty} \\
\leq & \| \sum_{q=0}^{m-1} h \int_{0}^{1} K\left(x, x_{q}+h \eta\right)\left[\varphi\left(x_{q}+h \eta\right)-\sum_{j=0}^{n} \frac{\varphi^{(j)}\left(x_{q}\right) h^{j} \eta^{j}}{j !}\right. \\
& \left.+\sum_{j=0}^{n} \frac{\varphi^{(j)}\left(x_{q}\right) h^{j} \eta^{j}}{j !}-\sum_{j=0}^{n} \frac{\varphi_{q}^{(j)} h^{j} \eta^{j}}{j !}\right] d \eta \|_{\infty} \\
\leq & \left\|\sum_{q=0}^{m-1} \frac{h^{n+2}}{(n+1) !} \int_{0}^{1} K\left(x, x_{q}+h \eta\right) \varphi^{(n+1)}\left(\theta_{q}\right) \eta^{n+1} d \eta\right\|_{\infty} \\
& +\left\|\sum_{q=0}^{m-1} \sum_{j=0}^{n} \frac{h^{j+1}}{j !}\left[\varphi^{(j)}\left(x_{q}\right)-\varphi_{q}^{(j)}\right] \int_{0}^{1} K\left(x, x_{q}+h \eta\right) \eta^{j} d \eta\right\|_{\infty} \\
\leq & \frac{(b-a) M N h^{n+1}}{(n+1) !}+h M \sum_{q=0}^{m-1} \sum_{j=0}^{n} \frac{h^{j}}{j !} \cdot\left\|\varphi^{(j)}\left(x_{q}\right)-\varphi_{q}^{(j)}\right\|_{\infty} \\
\leq & \frac{(b-a) M N h^{n+1}}{(n+1) !}+(b-a) M e^{h} \cdot \frac{N L h^{n+1} e^{b-a}[h+(b-a) M]}{\left[1-(b-a) M L e^{h+(b-a)}\right](n+1) !} \\
\left.\leq 1-(b-a) M L e^{h+(b-a)}\right] & \frac{h^{n+1}}{(n+1) !} \cdot
\end{aligned}
$$

In the end, we have

$$
\lim _{n \rightarrow+\infty}\left\|\varphi_{m, n}(x)-\varphi(x)\right\|_{\infty}=0
$$

and

$$
\lim _{h \rightarrow 0}\left\|\varphi_{m, n}(x)-\varphi(x)\right\|_{\infty}=0 .
$$

The proof is completed.

Similar to those in [29], a pair of feasible values for $m$ (i.e. $h$ ) and $n$ can be chosen to obtain a good approximation of the exact solution. The above observations will be further verified by using the numerical examples in the next section.

\section{Numerical Results}

In order to show the effectiveness of the proposed methods, we give several numerical examples. First, we compare the proposed method in the present paper with that in [29] and give Example 1. 
Table 1 The absolute errors of approximate and exact solutions for Example 1

\begin{tabular}{lllllll}
\hline$x$ & \multicolumn{1}{l}{$\left|\varphi(x)-\varphi_{m, n}(x)\right|$ for various pairs of $(m, n)$} & & & \\
\cline { 2 - 6 } & $(2,2)$ & $(2,3)$ & $(2,4)$ & $(4,2)$ & $(4,3)$ & $(4,4)$ \\
\hline 0.10 & $2.4409 \mathrm{e}-6$ & $5.3333 \mathrm{e}-8$ & $9.7388 \mathrm{e}-10$ & $2.3944 \mathrm{e}-7$ & $1.5073 \mathrm{e}-09$ & $2.9159 \mathrm{e}-12$ \\
0.20 & $9.4559 \mathrm{e}-6$ & $1.0982 \mathrm{e}-7$ & $5.2815 \mathrm{e}-10$ & $2.0862 \mathrm{e}-7$ & $2.2121 \mathrm{e}-09$ & $1.0288 \mathrm{e}-11$ \\
0.30 & $1.4172 \mathrm{e}-5$ & $6.0804 \mathrm{e}-8$ & $2.4506 \mathrm{e}-09$ & $7.9467 \mathrm{e}-8$ & $1.1769 \mathrm{e}-09$ & $1.0706 \mathrm{e}-11$ \\
0.40 & $8.1844 \mathrm{e}-6$ & $1.8787 \mathrm{e}-7$ & $1.7840 \mathrm{e}-09$ & $5.4620 \mathrm{e}-7$ & $8.9138 \mathrm{e}-10$ & $2.3890 \mathrm{e}-11$ \\
0.50 & $6.4122 \mathrm{e}-7$ & $2.2406 \mathrm{e}-9$ & $2.4513 \mathrm{e}-12$ & $1.9920 \mathrm{e}-8$ & $1.8241 \mathrm{e}-11$ & $4.5606 \mathrm{e}-15$ \\
0.60 & $4.5172 \mathrm{e}-6$ & $1.2861 \mathrm{e}-7$ & $2.3540 \mathrm{e}-09$ & $5.3349 \mathrm{e}-7$ & $3.6835 \mathrm{e}-09$ & $6.1419 \mathrm{e}-12$ \\
0.70 & $2.0468 \mathrm{e}-5$ & $2.6723 \mathrm{e}-7$ & $1.0004 \mathrm{e}-09$ & $4.7660 \mathrm{e}-7$ & $4.9360 \mathrm{e}-09$ & $2.2609 \mathrm{e}-11$ \\
0.80 & $3.1420 \mathrm{e}-5$ & $1.0660 \mathrm{e}-7$ & $5.4493 \mathrm{e}-09$ & $1.7008 \mathrm{e}-7$ & $2.5689 \mathrm{e}-09$ & $2.3686 \mathrm{e}-11$ \\
0.90 & $1.9112 \mathrm{e}-5$ & $3.9152 \mathrm{e}-7$ & $3.5898 \mathrm{e}-09$ & $1.1865 \mathrm{e}-6$ & $1.6643 \mathrm{e}-09$ & $5.0942 \mathrm{e}-11$ \\
1.00 & $6.4122 \mathrm{e}-7$ & $2.2406 \mathrm{e}-9$ & $2.4513 \mathrm{e}-12$ & $1.9920 \mathrm{e}-8$ & $1.8241 \mathrm{e}-11$ & $4.5607 \mathrm{e}-15$
\end{tabular}

Example 1 [29] A three-point boundary value problem for a second-order ordinary differential equation with variable coefficients is given as

$$
\left\{\begin{array}{l}
\varphi^{\prime \prime}(x)-(1+\sin x) \varphi(x)=-e^{x} \sin x \\
\varphi(0)=1, \quad \varphi(1)+\varphi(1 / 2)=e+e^{1 / 2}
\end{array}\right.
$$

with $x \in[0,1]$ and the exact solution $\varphi(x)=e^{x}$.

As shown in [29], the solution is unique and the approximate solution has been calculated. Here one computes the example again by using the present method and the results are given in Table 1. The parameters $m$ and $n$ are still chosen as $(m, n)=(2,2),(m, n)=(2,3)$, $(m, n)=(2,4),(m, n)=(4,2),(m, n)=(4,3)$ and $(m, n)=(4,4)$ respectively. As compared to the observations in Table 1 and those in [29], the results in Table 1 have more accuracy than those in [29] for the same pair parameters of $(m, n)$. It is further seen that the proposed method in the present study can be used not only to Fredholm integral equations of the second kind with continuous kernels, but also to those with weakly singular kernels. That is to say, the present method is more adaptable than that in [29]. To further show the effectiveness of the present method, the exact and the approximate solutions are plotted in Fig. 1 for $(m, n)=(1,0)$ with $y=\varphi(x)$ or $\varphi_{m, n}(x)$.

Second, we consider the generalized Bagley-Torvik equation with three-point boundary value conditions for $\alpha=3 / 2$ and give Example 2 .

Example 2 Assume that a three-point boundary value problem for the generalized BagleyTorvik equation is expressed as

$$
\left\{\begin{array}{l}
\varphi^{\prime \prime}(x)+\sqrt{\pi} x^{2} D^{\frac{3}{2}} \varphi(x)+\left(1-4 x^{\frac{1}{2}}\right) \varphi(x)=x^{2}+2 \\
\varphi(0)=0, \quad \varphi(1 / 5)+\varphi(1 / 10)=1 / 20
\end{array}\right.
$$

with $x \in\left[0, \frac{1}{5}\right]$ and the exact solution $\varphi(x)=x^{2}$.

Now we deal with the uniqueness of the solution of Example 2. It is calculated that

$$
|V(x, t)|=\left|(x-t)(1-4 \sqrt{t})-8 t \sqrt{x-t}+\frac{x^{2}}{\sqrt{x-t}}\right| .
$$




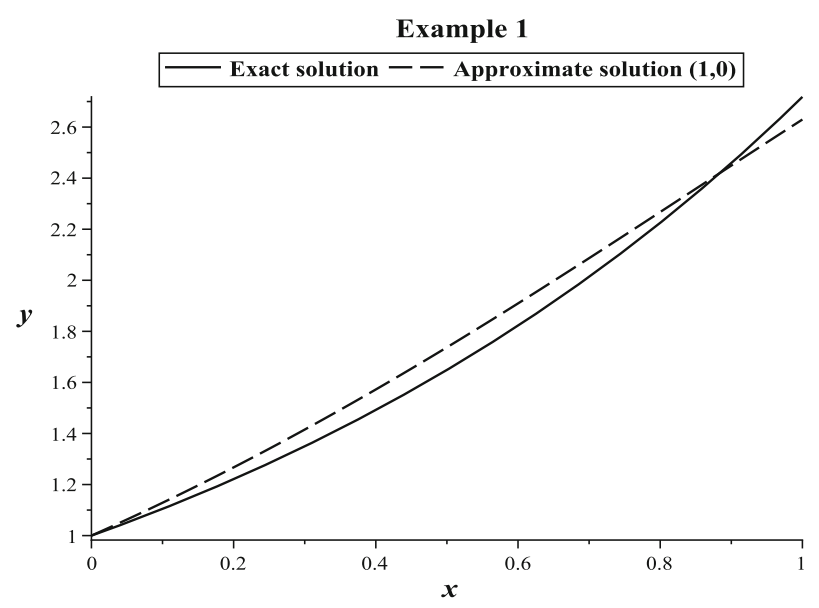

Fig. 1 The exact and the approximate solutions for $(m, n)=(1,0)$ with $y=\varphi(x)$ or $\varphi_{m, n}(x)$

According to Theorem 1 one has

$$
\begin{aligned}
& \max _{0 \leq x \leq \frac{1}{5}} \int_{0}^{\frac{1}{5}}\left|K_{1}(x, t)\right| d t \\
& =\max _{0 \leq x \leq \frac{1}{5}} \int_{0}^{x}|V(x, t)| d t+\frac{10 x}{3} \int_{0}^{\frac{1}{5}}\left|V\left(\frac{1}{5}, t\right)\right| d t+\frac{10 x}{3} \int_{0}^{\frac{1}{10}}\left|V\left(\frac{1}{10}, t\right)\right| d t \\
& \leq \max _{0 \leq x \leq \frac{1}{5}}\left[\frac{26}{5} x^{\frac{5}{2}}+\frac{1}{2} x^{2}+\left(\frac{1}{12}+\frac{52 \sqrt{5}}{375}+\frac{13 \sqrt{10}}{750}\right) x\right] \approx 0.2027<1 .
\end{aligned}
$$

It is seen that the boundary value problem has a unique solution by using Theorem 3 . Moreover, the absolute errors of the approximate and exact solutions are shown in Table 2. One can find from Table 2 that a good approximation has been obtained by using the proposed method. As shown in Fig. 2, the absolute errors between the approximate and exact solutions are very small even for $(m, n)=(1,0)$.

Third, we consider $\alpha=1 / 2$ and give Example 3 .

Example 3 A three-point boundary value problem for the fractional Bagley-Torvik equation with variable coefficients is written as

$$
\left\{\begin{array}{l}
\varphi^{\prime \prime}(x)-5 \sqrt{\pi} x D^{\frac{1}{2}} \varphi(x)+16 x^{\frac{1}{2}} \varphi(x)=6 x \\
\varphi(0)+2 \varphi(1 / 10)=1 / 500, \quad \varphi(1 / 5)=1 / 125,
\end{array}\right.
$$

with $x \in\left[0, \frac{1}{5}\right]$ and the exact solution $\varphi(x)=x^{3}$.

Now we deal with the uniqueness of the solution of Example 3. It is calculated that

$$
|V(x, t)|=\left|16 \sqrt{t}(x-t)+\frac{10}{3}(x-4 t) \sqrt{x-t}\right|,
$$

and

$$
|\Lambda(x, t)|=\left|\frac{10}{3}(x-4 t) \sqrt{x-t}+\frac{2}{3}(20 t-15 x+1) \sqrt{\frac{1}{10}-t}+\frac{1}{20} \frac{10 x-1}{\sqrt{\frac{1}{10}-t}}\right| .
$$




\section{Example 2}

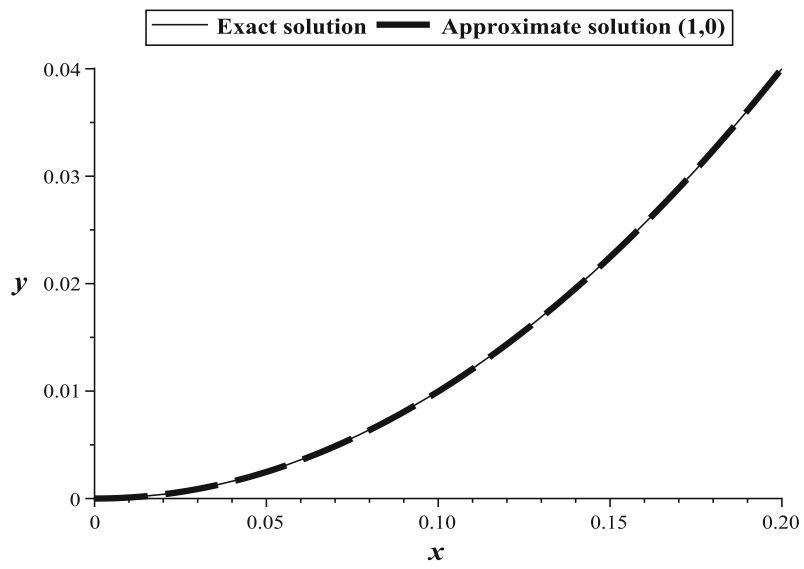

Fig. 2 The exact and the approximate solutions for $(m, n)=(1,0)$ with $y=\varphi(x)$ or $\varphi_{m, n}(x)$

Table 2 The absolute errors of approximate and exact solutions for Example 2

\begin{tabular}{lllllll}
\hline$x$ & \multicolumn{6}{l}{$\left|\varphi(x)-\varphi_{m, n}(x)\right|$ for various pairs of $(m, n)$} \\
& \cline { 2 - 6 } & $(2,0)$ & $(4,0)$ & $(4,1)$ & $(8,0)$ & $(8,1)$ \\
\hline 0.02 & $9.8220 \mathrm{e}-6$ & $1.7560 \mathrm{e}-6$ & $4.8489 \mathrm{e}-6$ & $3.3231 \mathrm{e}-7$ & $2.0006 \mathrm{e}-6$ & $6.0631 \mathrm{e}-8$ \\
0.04 & $1.9457 \mathrm{e}-5$ & $3.6174 \mathrm{e}-6$ & $9.5112 \mathrm{e}-6$ & $6.2413 \mathrm{e}-7$ & $3.8910 \mathrm{e}-6$ & $1.3845 \mathrm{e}-7$ \\
0.06 & $2.8426 \mathrm{e}-5$ & $5.2283 \mathrm{e}-6$ & $1.4591 \mathrm{e}-5$ & $1.1829 \mathrm{e}-6$ & $5.8598 \mathrm{e}-6$ & $2.2686 \mathrm{e}-7$ \\
0.08 & $3.5928 \mathrm{e}-5$ & $5.7881 \mathrm{e}-6$ & $1.7301 \mathrm{e}-5$ & $1.6487 \mathrm{e}-6$ & $7.9866 \mathrm{e}-6$ & $2.7619 \mathrm{e}-7$ \\
0.10 & $4.0843 \mathrm{e}-5$ & $4.0770 \mathrm{e}-6$ & $1.6630 \mathrm{e}-5$ & $7.4029 \mathrm{e}-7$ & $6.2251 \mathrm{e}-6$ & $1.3286 \mathrm{e}-7$ \\
0.12 & $6.5460 \mathrm{e}-5$ & $1.4499 \mathrm{e}-5$ & $2.9073 \mathrm{e}-5$ & $2.9104 \mathrm{e}-6$ & $7.9716 \mathrm{e}-6$ & $3.7253 \mathrm{e}-7$ \\
0.14 & $6.2870 \mathrm{e}-5$ & $1.9870 \mathrm{e}-5$ & $1.8837 \mathrm{e}-5$ & $2.2855 \mathrm{e}-6$ & $1.1839 \mathrm{e}-5$ & $6.1163 \mathrm{e}-7$ \\
0.16 & $4.5301 \mathrm{e}-5$ & $2.0809 \mathrm{e}-5$ & $4.1940 \mathrm{e}-5$ & $3.2812 \mathrm{e}-6$ & $1.7801 \mathrm{e}-5$ & $7.3445 \mathrm{e}-7$ \\
0.18 & $7.1547 \mathrm{e}-5$ & $1.4117 \mathrm{e}-5$ & $3.5404 \mathrm{e}-5$ & $4.6672 \mathrm{e}-6$ & $2.2642 \mathrm{e}-5$ & $6.3016 \mathrm{e}-7$ \\
0.20 & $4.0843 \mathrm{e}-5$ & $4.0770 \mathrm{e}-6$ & $1.6630 \mathrm{e}-5$ & $7.4029 \mathrm{e}-7$ & $6.2251 \mathrm{e}-6$ & $1.3286 \mathrm{e}-7$ \\
\hline
\end{tabular}

According to Theorem 2 one has

$$
\begin{aligned}
& \max _{0 \leq x \leq \frac{1}{5}} \int_{0}^{\frac{1}{5}}\left|K_{2}(x, t)\right| d t \\
& \leq \max _{0 \leq x \leq \frac{1}{5}} \frac{1}{2} \int_{0}^{x}|V(x, t)| d t+\frac{5 x}{2} \int_{0}^{\frac{1}{5}}\left|V\left(\frac{1}{5}, t\right)\right| d t+\frac{1}{2} \int_{\frac{1}{10}}^{x}|V(x, t)| d t \\
& \quad+5\left(x+\frac{1}{10}\right) \int_{\frac{1}{10}}^{\frac{1}{5}}\left|V\left(\frac{1}{5}, t\right)\right| d t+\frac{1}{2} \int_{0}^{\frac{1}{10}}|\Lambda(x, t)| d t \\
& \quad+5\left(x+\frac{1}{10}\right) \int_{0}^{\frac{1}{10}}\left|\Lambda\left(\frac{1}{5}, t\right)\right| d t \\
& \approx 0.7693<1 .
\end{aligned}
$$




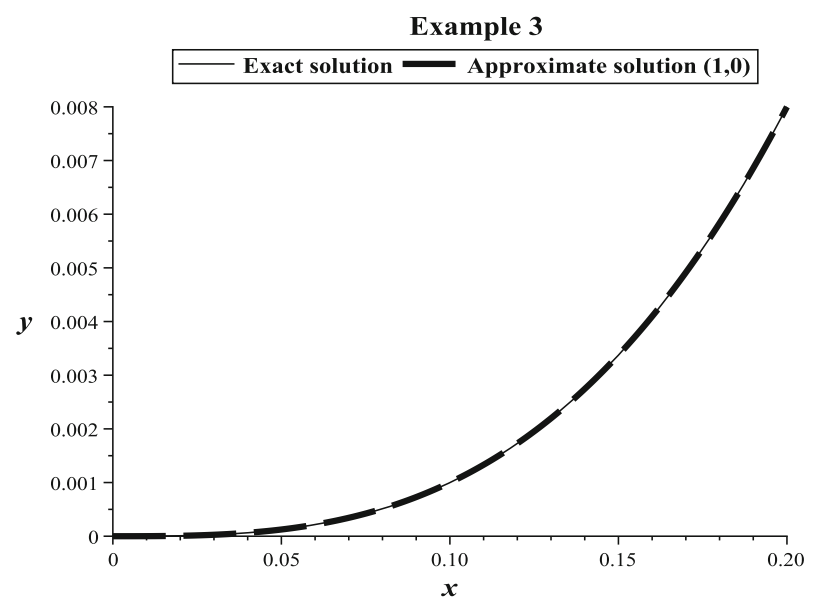

Fig. 3 The exact and the approximate solutions for $(m, n)=(1,0)$ with $y=\varphi(x)$ or $\varphi_{m, n}(x)$

Table 3 The absolute errors of approximate and exact solutions for Example 3

\begin{tabular}{lllllll}
\hline$x$ & \multicolumn{6}{l}{$\left|\varphi(x)-\varphi_{m, n}(x)\right|$ for various pairs of $(m, n)$} \\
\cline { 2 - 7 } & $(2,0)$ & $(2,1)$ & $(2,2)$ & $(4,0)$ & $(4,1)$ & $(4,2)$ \\
\hline 0.00 & $1.8750 \mathrm{e}-6$ & $1.6321 \mathrm{e}-6$ & $3.3402 \mathrm{e}-9$ & $1.3150 \mathrm{e}-6$ & $1.9344 \mathrm{e}-7$ & $8.1180 \mathrm{e}-11$ \\
0.02 & $1.3157 \mathrm{e}-6$ & $9.3094 \mathrm{e}-7$ & $2.0659 \mathrm{e}-9$ & $9.3937 \mathrm{e}-7$ & $1.1255 \mathrm{e}-7$ & $2.5205 \mathrm{e}-10$ \\
0.04 & $7.5519 \mathrm{e}-7$ & $2.6518 \mathrm{e}-7$ & $1.1401 \mathrm{e}-8$ & $5.6299 \mathrm{e}-7$ & $4.0605 \mathrm{e}-8$ & $1.2628 \mathrm{e}-09$ \\
0.06 & $3.9308 \mathrm{e}-7$ & $1.0391 \mathrm{e}-7$ & $3.9217 \mathrm{e}-8$ & $2.0555 \mathrm{e}-7$ & $2.7457 \mathrm{e}-8$ & $1.1225 \mathrm{e}-09$ \\
0.08 & $6.7104 \mathrm{e}-7$ & $3.9608 \mathrm{e}-7$ & $5.6790 \mathrm{e}-8$ & $1.7126 \mathrm{e}-7$ & $1.1906 \mathrm{e}-7$ & $3.7779 \mathrm{e}-10$ \\
0.10 & $1.3749 \mathrm{e}-6$ & $8.1603 \mathrm{e}-7$ & $1.6701 \mathrm{e}-9$ & $6.5748 \mathrm{e}-7$ & $9.6719 \mathrm{e}-8$ & $4.0590 \mathrm{e}-11$ \\
0.12 & $2.4253 \mathrm{e}-6$ & $1.4781 \mathrm{e}-6$ & $5.1651 \mathrm{e}-8$ & $4.8432 \mathrm{e}-7$ & $1.8599 \mathrm{e}-7$ & $3.4848 \mathrm{e}-09$ \\
0.14 & $3.5371 \mathrm{e}-6$ & $2.5651 \mathrm{e}-6$ & $7.0735 \mathrm{e}-8$ & $8.0560 \mathrm{e}-7$ & $2.5485 \mathrm{e}-7$ & $1.4666 \mathrm{e}-09$ \\
0.16 & $3.6703 \mathrm{e}-6$ & $3.3204 \mathrm{e}-6$ & $1.8646 \mathrm{e}-8$ & $5.0344 \mathrm{e}-7$ & $7.7106 \mathrm{e}-8$ & $3.3408 \mathrm{e}-09$ \\
0.18 & $3.5553 \mathrm{e}-6$ & $2.8250 \mathrm{e}-6$ & $6.3534 \mathrm{e}-8$ & $1.1779 \mathrm{e}-6$ & $3.0832 \mathrm{e}-7$ & $3.5001 \mathrm{e}-09$
\end{tabular}

So the boundary value problem also has a unique solution by using Theorem 4 . Furthermore, the numerical results are carried out and shown in Fig. 3 and Table 3 by using the present method. As shown in Tables 1, 2 and 3, one can see that when $m$ or $n$ is increasing, the absolute errors $\left|\varphi(x)-\varphi_{m, n}(x)\right|$ is decreasing. The obtained results are in good agreement with the theoretical analysis in Theorem 5 .

\section{Conclusions}

To simulate a real case, the Bagley-Torvik equation is generalized by equipping variable coefficients and three-point boundary conditions. The integration method is proposed to transform the three point boundary value problems into Fredholm integral equations of the second kind. Different from those for ordinary differential equations, the obtained Fredholm 
integral equations of the second kind may be with weakly singular kernels depending on the fractional order and boundary value conditions. A new numerical method for Fredholm integral equations of the second kind with weakly singular kernels has been further proposed. The approximate solution has been constructed, and its convergence and error estimate have been made. Numerical results have been shown that the proposed solution procedures are effective.

Acknowledgments The authors would like to thank the reviewer for the valuable suggestions and comments of improving the paper. The work was supported by the National Natural Science Foundation of China (No: 11362002), the project of outstanding young teachers' training in higher education institutions of Guangxi, and the project of Guangxi Colleges and Universities Key Laboratory of Mathematics and Its Applications.

\section{References}

1. Ortigueira, M.D.: Fractional Calculus for Scientists and Engineers. Springer, New York (2011)

2. Teodor, M.A., Pilipovic, S.: Fractional Calculus with Applications in Mechanics: Vibrations and Diffusion Processes. ISTE Ltd and John Wiley and Sons Inc, Hoboken (2014)

3. Uchaikin, V.V.: Fractional Derivatives for Physicists and Engineers. Higher Education Press, Beijing (2013)

4. Torvik, P.J., Bagley, R.L.: On the appearance of the fractional derivative in the behavior of real materials. ASME Trans. J. Appl. Mech. 51, 294-298 (1984)

5. Diethelm, K., Ford, J.: Numerical solution of the Bagley-Torvik equation. BIT Numer. Math. 42(3), 490-507 (2002)

6. Ray, S.S., Bera, R.K.: Analytical solution of the Bagley-Torvik equation by Adomian decomposition method. Appl. Math. Comput. 168, 398-410 (2005)

7. Zolfaghari, M., Ghaderi, R., Sheikhol-Eslami, A., et al.: Application of the enhanced homotopy perturbation method to solve the fractional-order Bagley-Torvik differential equation. Phys. Scr. T136, 014032 (2009)

8. Wang, Z.H., Wang, X.: General solution of the Bagley-Torvik equation with fractional-order derivative. Commun. Nonlinear Sci. Numer. Simulat. 15, 1279-1285 (2010)

9. Cenesiz, Y., Keskin, Y., Kurnaz, A.: The solution of the Bagley-Torvik equation with the generalized Taylor collocation method. J. Frankl. Inst. 347, 452-466 (2010)

10. Raja, M.A.Z., Khan, J.A., Qureshi, I.M.: Solution of fractional order system of Bagley-Torvik equation using evolutionary computational intelligence. Math. Probl. Eng. 2011, 675075 (2011)

11. S, Saha Ray: On Haar wavelet operational matrix of general order and its application for the numerical solution of fractional Bagley-Torvik equation. Appl. Math. Comput. 218, 5239-5248 (2012)

12. Mekkaoui, T., Hammouch, Z.: Approximate analytical solutions to the Bagley-Torvik equation by the fractional iteration method. An. Univ. Craiova Ser. Mat. Inform. 39(2), 251-256 (2012)

13. Atanackovic, T.M., Zorica, D.: On the Bagley-Torvik equation. ASME J. Appl. Mech. 80, 041013 (2013)

14. Setia, A., Liu, Y.C., Vatsala, A.S.: The solution of the Bagley-Torvik equation by using second kind Chebyshev wavelet. IEEE 11th International Conference on Information Technology: New Generations, pp. 443-446 (2014)

15. Cermak, J., Kisel, T.: Exact and discretized stability of the Bagley-Torvik equation. J. Comput. Appl. Math. 269, 53-67 (2014)

16. Stanek, S.: Two-point boundary value problems for the generalized Bagley-Torvik fractional differential equation. Cent. Eur. J. Math. 11(3), 574-593 (2013)

17. Keshavarz, E., Ordokhani, Y., Razzaghi, M.: Bernoulli wavelet operational matrix of fractional order integration and its applications in solving the fractional order differential equations. Appl. Math. Model. 38, 6038-6051 (2014)

18. Lubich, C.: Discretized fractional calculus. SIAM J. Math. Anal. 17, 704-719 (1986)

19. Podlubny, I.: Fractional Differential Equations. Academic Press, New York (1993)

20. Kazem, S., Abbasbandy, S., Kumar, S.: Fractional-order Legendre functions for solving fractional-order differential equations. Appl. Math. Model. 37, 5498-5510 (2013)

21. Diethelm, K.: Generalized compound quadrature formulae for finite-part integrals. IMA J. Numer. Anal. 17, 479-493 (1997)

22. Diethelm, K., Ford, N.J., Freed, A.D., Luchko, Y.: Algorithms for the fractional calculus: a selection of numerical methods. Comput. Methods Appl. Mech. Eng. 194, 743-773 (2005) 
23. Lopez-Fernandez, M., Lubich, C., Schadle, A.: Adaptive, fast, and oblivious convolution in evolution equations with memory. SIAM J. Sci. Comput. 30, 1015-1037 (2008)

24. Ma, X.H., Huang, C.M.: Spectral collocation method for linear fractional integro-differential equations. Appl. Math. Model. 38, 1434-1448 (2014)

25. Li, J.R.: A fast time stepping method for evaluating fractional integrals. SIAM J. Sci. Comput. 31, 46964714 (2010)

26. Bai, Z.B.: On positive solutions of a nonlocal fractional boundary value problem. Nonlinear Anal. 72, 916-924 (2010)

27. Jia, Y.L., Zhang, X.Q.: Positive solutions for a class of fractional differential equation multi-point boundary value problems with changing sign nonlinearity. J. Appl. Math. Comput. 47, 15-31 (2015)

28. Ahmad, B., Ntouyas, S.K.: On Hadamard fractional integro-differential boundary value problems. J. Appl. Math. Comput. 47, 119-131 (2015)

29. Zhong, X.C., Huang, Q.A.: Approximate solution of three-point boundary value problems for secondorder ordinary differential equations with variable coefficients. Appl. Math. Comput. 247, 18-29 (2014)

30. Richter, G.R.: On weakly singular Fredholm integral equations with displacement kernels. J. Math. Anal. Appl. 55, 32-42 (1976)

31. Goursat, E.: A Course in Mathematical Analysis. Part 2: Integral Equations, Calculus of Variations, vol. III. Dover, New York (1964)

32. Lang, S.: Real and Functional Analysis, 3rd edn. Springer, New York (1993) 